OPEN ACCESS

Edited by:

Zhanglin Lin,

South China University of Technology,

China

Reviewed by:

Yixin Huo,

Beijing Institute of Technology, China

Mario Andrea Marchisio,

Tianjin University, China

*Correspondence:

Cheng Lu

lucheng@swu.edu.cn;

lucheng@sue.edu.cn

Minhui Pan

pmh047@126.com

${ }^{\dagger}$ These authors have contributed equally to this work

Specialty section: This article was submitted to

Synthetic Biology,

a section of the journal Frontiers in Bioengineering and

Biotechnology

Received: 20 April 2020

Accepted: 30 June 2020

Published: 15 July 2020

Citation:

Dong Z, Qin Q, Hu Z, Zhang X, Miao J, Huang L, Chen P, LU C and Pan M (2020) CRISPR/Cas12a Mediated Genome Editing Enhances Bombyx mori Resistance to BmNPV.

Front. Bioeng. Biotechnol. 8:841.

doi: 10.3389/fbioe.2020.00841

\section{CRISPR/Cas12a Mediated Genome Editing Enhances Bombyx mori Resistance to BmNPV}

\author{
Zhanqi Dong't, Qi Qin ${ }^{1 \dagger}$, Zhigang Hu ${ }^{1}$, Xinling Zhang ${ }^{1}$, Jianghao Miao', Liang Huang', \\ Peng Chen ${ }^{1}$, Cheng $\mathrm{Lu}^{1,2 *}$ and Minhui Pan ${ }^{1,2 *}$ \\ 'State Key Laboratory of Silkworm Genome Biology, Southwest University, Chongqing, China, ${ }^{2}$ Key Laboratory \\ of Sericultural Biology and Genetic Breeding, Ministry of Agriculture and Rural Affairs, Southwest University, Chongqing, \\ China
}

CRISPR/Cas12a (Cpf1) is a single RNA-guided endonuclease that provides new opportunities for targeted genome engineering through the CRISPR/Cas9 system. Only AsCas12a has been developed for insect genome editing, and the novel Cas12a orthologs nucleases and editing efficiency require more study on insects. We compared three Cas12a orthologs nucleases, AsCas12a, FnCas12a, and LbCas12a, for their editing efficiencies and antiviral abilities. The three Cas12a efficiently edited the Bombyx mori nucleopolyhedrovirus (BmNPV) genome and inhibited BmNPV replication in BmNSWU1 cells. The antiviral ability of the FnCas12a system was more efficient than that of the SpCas9 system after infection by BmNPV. We created FnCas12a $\times$ glE1 and SpCas $9 \times$ sglE1 transgenic hybrid lines and evaluated the gene-editing efficiency of different systems at the same target site. We improved the antiviral ability using the FnCas12a system in transgenic silkworm. This study demonstrated the use of the CRISPR/Cas12a system to achieve high editing efficiencies, and increase disease resistance in the silkworm.

Keywords: CRISPR/Cas12a, genome editing, antiviral therapy, Bombyx mori, BmNPV

\section{INTRODUCTION}

Genome editing introduces DNA mutations in the form of insertions, deletions or base substitutions within selected DNA sequences (Backes et al., 2018). Clustered regularly interspaced short palindromic repeats (CRISPR) gene-editing technology has been used in gene function research, genetic improvement, modeling biology and gene therapy (Burgess, 2013; AlvesBezerra et al., 2019; Beretta and Mouquet, 2019; Li et al., 2019). Three effector proteins 
of class 2 type $\mathrm{V}$ CRISPR systems, the CRISPR/CRISPRassociated 12a (Cas12a, known as Cpf1) proteins of Lachnospiraceae bacterium (LbCas12a), Francisella novicida (FnCas12a), and Acidaminococcus sp. (AsCas12a), have been shown to efficiently edit mammalian cell genomes with more efficient genome editing than the widely used Streptococcus pyogenes CRISPR/CRISPR-associated 9 (SpCas9) (Fagerlund et al., 2015; Zetsche et al., 2015; Gao et al., 2017). However, the CRISPR/Cas12a system has rarely been used for insect genome editing research and antiviral therapy (Ma et al., 2017).

The silk industry suffers great economic losses due to $B$. mori nucleopolyhedrovirus (BmNPV) infection (Isobe et al., 2004; Subbaiah et al., 2013; Dong et al., 2018a). CRISPR genome editing is an efficient and widely used technology for antiBmNPV gene therapy, viral gene function research, and screening of potential targets in BmNPV infection (Dong et al., 2018a,b; Dong et al., 2018b). We first reported on a highly efficient virus-inducible gene-editing system, which demonstrated that CRISPR/Cas9 could edit the BmNPV genome and effectively inhibit virus proliferation (Dong Z.Q. et al., 2016). Chen et al. (2017) effectively inhibited BmNPV proliferation and replication by editing the $i e-1$ and me53 of BmNPV immediate early genes in transgenic silkworm. We improved the antiviral ability of transgenic silkworm nearly 1,000-fold by editing the two target sites of the ie-1 gene to produce a large fragment deletion (Dong et al., 2018a). The CRISPR/Cas9 gene-editing technology has also been used in antiviral resistance breeding by editing host factors and viral key genes in BmNPV infection. However, the antiviral resistance level using this system has reached a plateau (Dong et al., 2018b, 2019a,b).

The CRISPR/Cas12a system (Cpf1) is a single RNA-guided endonuclease used for genome editing (Fagerlund et al., 2015). The Cas12a enzyme has several gene-editing characteristics that differ from the Cas9 system (Zetsche et al., 2015; Dong D. et al., 2016). One major difference between the Cas12a and Cas9 systems is that Cas12a recognizes a T-rich protospacer-adjacent motif (PAM), while Cas9 recognizes a G-rich PAM (Fonfara et al., 2016). The Cas12a system increases number of the potential target sites that can be used for CRISPR-mediated gene-editing (Fonfara et al., 2016). Cas12a enzyme requires one U6 (Pol-III) promoter to drive small CRISPR-derived RNA (crRNAs, 42-44nt per-crRNA, 19-nt repeat and 23-25-nt spacer). However, the Cas9 enzyme requires a single guide RNA (sgRNA) derived from the fusion of CRISPR RNA (crRNA) and trans-activating crRNA (tracrRNA) (Burgess, 2013; Fonfara et al., 2016). Multiple crRNAs can be expressed as a single transcript to generate functional individual crRNAs after processing through Cas12a nuclease; this can increase the efficiency of crRNA entry into cells (Fagerlund et al., 2015; Nakade et al., 2017). Cas12a nuclease also generates a 5-bp staggered DNA double-strand break ends that are formed downstream of the PAM sequence, while Cas9 nuclease only formed a blunt-end cut 3 bp upstream of the PAM sequence (Mahfouz, 2017; Nakade et al., 2017). The unique editing features of the Cas12a system are conducive to overcoming the limitations of the Cas9 system.

We investigated the ability of AsCas12a, FnCas12a, and LbCas12a to edit BmNPV genomes in B. mori. Our goals were to compare the gene-editing efficiency of the Cas12a and Cas9 systems in anti-BmNPV therapy and to develop transgenic silkworms with BmNPV resistance. Initially, an AsCas12a, FnCas12a and LbCas12a-based gene-editing vector and the crRNA expression cassette were developed. Then, different Cas12a nuclease activities with crRNA derived by the U6 promoter were evaluated for gene-editing efficiency and antiviral ability in BmN-SWU1 cells. The antiviral abilities of FnCas12a and SpCas9 systems, which are widely used for BmNPV genome editing, were compared. Finally, the gene-editing efficiency and resistance level of the transgenic FnCas12a and SpCas9 lines were evaluated by mortality analyses, sequencing and viral gene transcription in transgenic silkworms.

\section{RESULTS}

\section{CRISPR/Cas12a System Can Edit the BmNPV Genome}

To determine whether the CRISPR/Cas12a system could be used for gene-editing in silkworm, we examined the functionality of three Cas12a enzymes, AsCas12a, FnCas12a, and LbCas12a, which have been used to edit the genomes of mammal cells. We constructed the expression cassettes of AsCas12a, FnCas12a, and LbCas12a, and connected them with the nuclear localization signal and $3 \times \mathrm{HA}$ tag. The expression cassette was initiated by the OpIE2 promoter and terminated by the OpIE2-PA. The crRNA expression cassettes consisting of a 20-21-nt direct repeat and a 23-nt guide sequence were arranged in tandem and driven by a signal U6 promoter of $B$. mori. Then, we transfected BmN-SWU1 cells with individual Cas12a orthologs and gRNA to target endogenous loci containing the $5^{\prime}$ T-rich PAMs (Figure 1A).

Editing the BmNPV ie-1 gene can effectively inhibit viral replication. We selected the $i e-1$ gene as a target for further analysis. To facilitate the quantification and comparison of these nucleases, we constructed one vector system containing Cas12a orthologs and gIE1. After transfecting the Cas12a system in BmN-SWU1 cells, Sanger sequencing analysis revealed that all of the Cas12a enzymes could edit the target site of the ie-1 gene. To analyze the gene-editing efficiency of different AsCas12a, FnCas12a, and LbCas12a systems, the genome of each group was extracted and $\mathrm{T}$ cloning was performed; 20 samples were randomly selected to sequence, and statistical analysis of editing efficiency of the putative cleavage site reached 22.0, 27.5, and $22.0 \%$, respectively (Figure 1B). To further analyze whether the Cas12a system could inhibit virus proliferation, we examined the change in VP39 protein expression after BmNPV infection. Western blot results showed that the expression of VP39 protein was significantly affected by eliminating the viral genome at $48 \mathrm{~h}$ post-infection (h p.i.). The VP39 protein expression levels of AsCas12a, FnCas12a, and LbCas12a systems were equivalent to $74.0,56.0$, and $61.0 \%$ of the control, respectively (Figure 1C). To demonstrate the antiviral efficiency of the CRISPR/Cas12a system, we also determined the replication of the viral genome through quantitative polymerase chain 


\section{A Cas12a Expression}

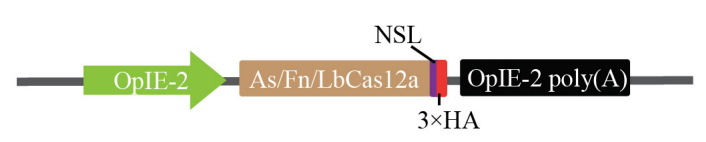

Guide RNA Expression
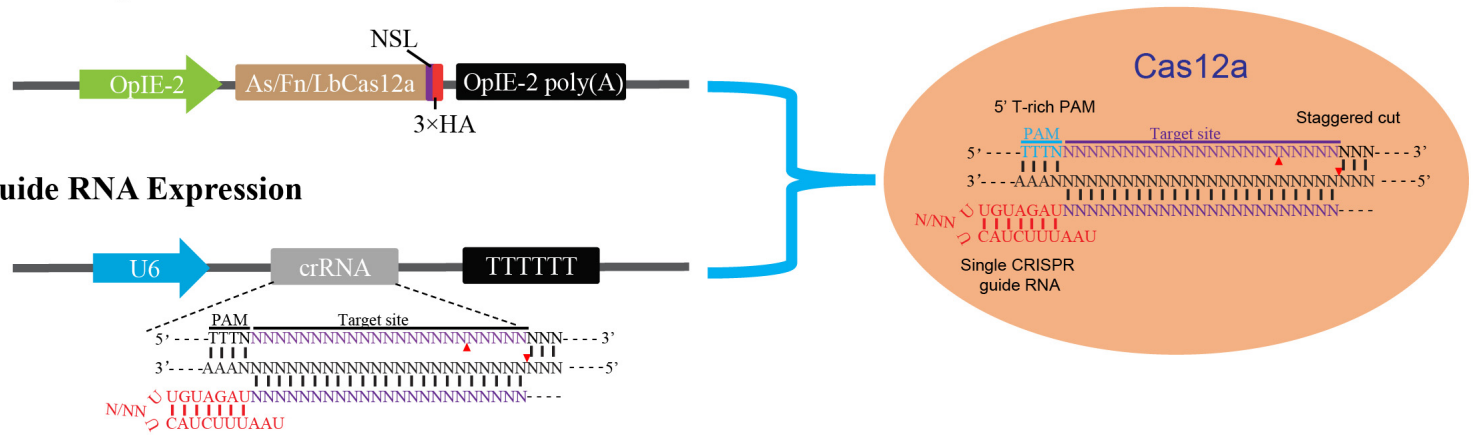

B

PAM Target site

WT 5'-CGGAATCTTTTGAGCAGTCTGTTGGTGTGAACCAACCATCGGCAGCTGGAACTAAACGGAAGCTGG-3'

Deletion 5'-CGGAATCTTTTGAGCAGTCTGTTGG - - TGAACCAACCATCGGCAGCTGGAACTAAACGGAAGCTGG-3' (2)

Deletion 5'-CGgAATCTTTTGAGCAGTCTGTTGGT - TGAACCAACCATCGGCAGCTGGAACTAAACGGAAGCTGG-3' (3)

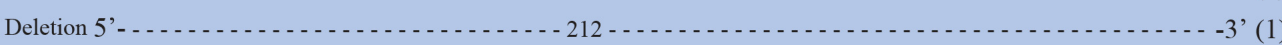

Deletion 5'-CGGAATCTTTTGAGCAGT - . . . . . . . . . . ACCAACCATCGGCAGCTGGAACTAAACGGAAGCTGG-3' (3)

AsCas12a

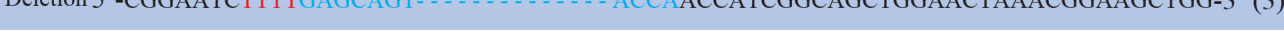

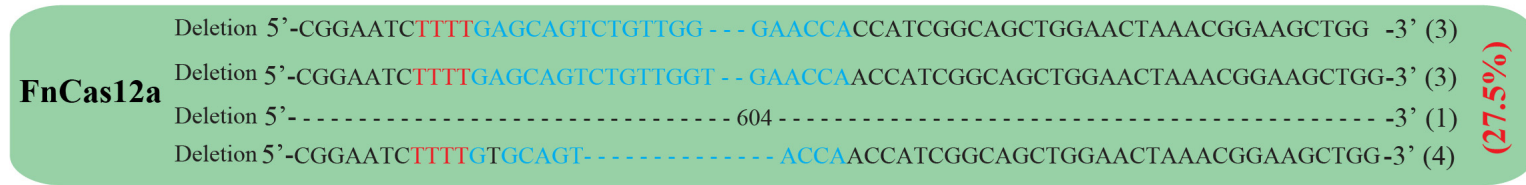

Deletion 5'-CGGAATCTTTTGAGCAGTCTGTTGG - - TGAACCAACCATCGGCAGCTGGAACTAAACGGAAGCTGG-3' (2)

LbCas12a

Deletion 5'-CGGAATCTTTTGAGCAGTCTGTT - . - . - TGAACCAACCATCGGCAGCTGGAACTAAACGGAAGCTGG-3' (4)

Deletion 5'-CGGAATCTTTTGAGCAGT- . . . . . . . . . . . . ACCAACCATCGGCAGCTGGAACTAAACGGAAGCTGG-3' (4)

Deletion 5'-CGGAATCTTTTGAGCAGT - . . . . . . . . - TGAACCAACCATCGGCAGCTGGAACTAAACGGAAGCTGG-3' (1)

C

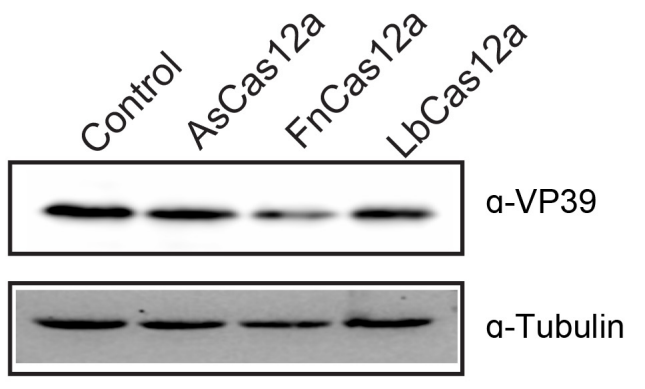

D

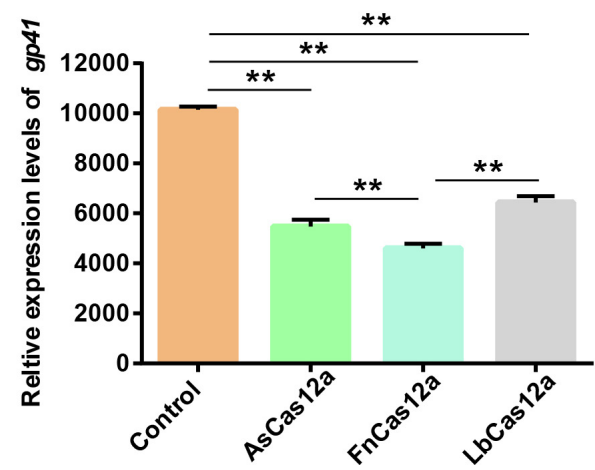

FIGURE 1 | CRISPR/Cas12a system enables BmNPV genome editing. (A) Cas12a expression cassette and crRNA target gene site. (B) DNA sequencing analysis of high-frequency genome mutations by Cas12a in BmN-SWU1 cells. The BmNPV ie-1 gene sequence is shown in black on top, the target site of gRNA is in blue, PAM sequence is in red, and the deletion sequence is indicated by dashes. (C) Western blot analysis of CRISPR/Cas12a system mediated antiviral activity monitored by the levels of the VP39 (top) and Tubulin (bottom). (D) DNA replication analysis of CRISPR/Cas12a system-mediated antiviral activity monitored by the copies of gp41. Error bars represent standard deviations of three biological replicates. ${ }^{* \star}$ represents a statistically significant difference at $P<0.01$.

reaction (qPCR) analysis. The amount of BmNPV DNA was affected after eliminating the viral genome. Compared with the control group, the AsCas12a, FnCas12a, and LbCas12a systems reduced BmNPV DNA by 46.0, 54.6, and $36.5 \%$, respectively (Figure 1D). Statistical analysis also showed that the FnCas12a system also has a significant decrease compared to the AsCas12a and LbCas12a systems (Figure 1D). All of the three constructed CRISPR/Cas12a gene-editing systems significantly 
A

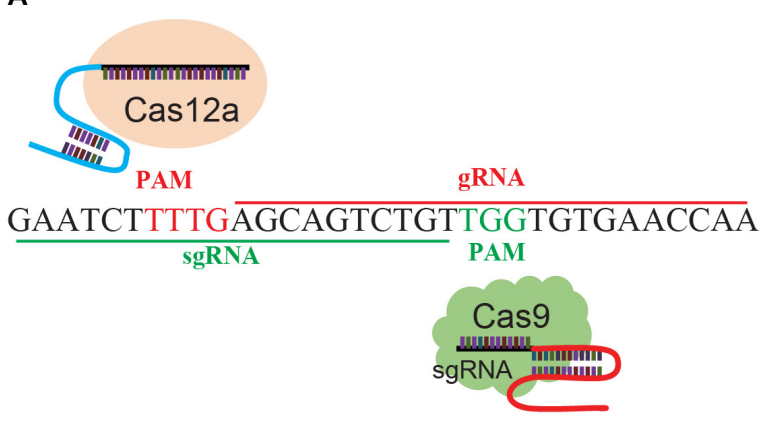

B

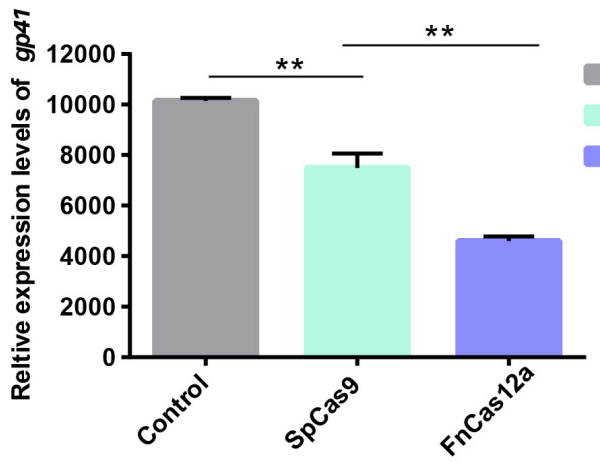

Control

SpCas9

FnCas12a

C

Control

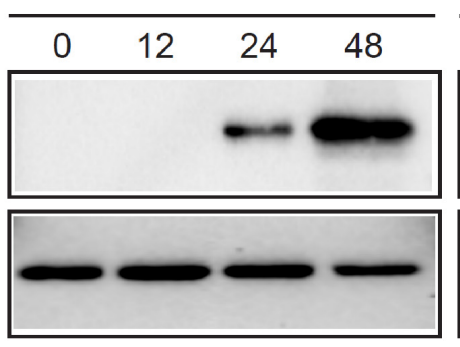

SpCas9

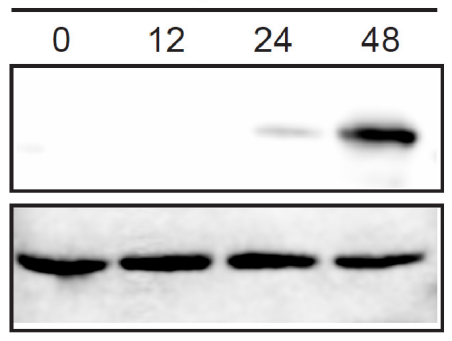

FnCas12a

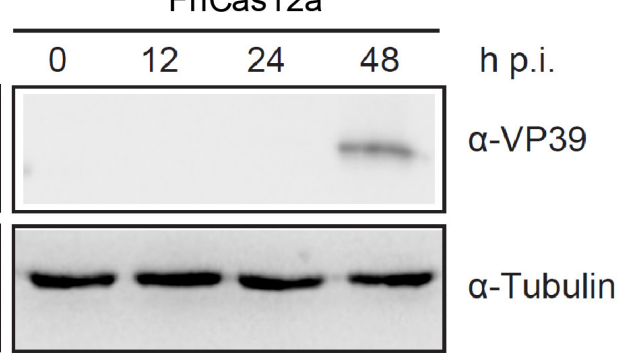

FIGURE 2 | Comparative analysis of the antiviral ability of CRISPR/Cas9 and CRISPR/Cas12a. (A) Comparison of CRISPR/Cas9 versus CRISPR/Cas12a-mediated genome editing. Cas12a, Cas9, crRNA, and PAM are shown. (B) Analysis of BmNPV DNA replication in CRISPR/Cas12a and CRISPR/Cas9 systems. Error bars represent standard deviations of three biological replicates. ${ }^{\star *}$ represents statistically significant differences at $P<0.01$. (C) Western blot analysis of CRISPR/Cas $12 a$ and CRISPR/Cas9 system mediated antiviral activity monitored by the levels of the VP39 (top) and Tubulin (bottom). The ratios of different types of mutations.

inhibited virus replication in B. mori, and the FnCas12a system had the greatest antiviral effect.

\section{Analysis of the Antiviral Ability of CRISPR/Cas9 and CRISPR/Cas12a}

To evaluate the performance of the different CRISPR systems in B. mori, we focused on FnCas12a and SpCas9 gene-editing systems for the same target site (Figure 2A). We initially chose the BmNPV ie-1 gene as the target. PAM profiling of FnCas12a and SpCas9 is shown in Figure 2A. After transfected with FnCas12a $\times$ gIE1 and SpCas $9 \times$ sgIE1 in BmN-SWU1 cells, the cells infected with the $\mathrm{vA} 4^{\mathrm{prm}}$-EGFP virus at multiplicity of infection (MOI) of 10. At $48 \mathrm{~h}$ p.i., viral DNA replication showed that different gene-editing systems could significantly inhibit BmNPV DNA replication, and the FnCas12a system had a greater inhibition effect compared with the SpCas9 system (Figure 2B). Compared to the control, BmNPV DNA replication levels were reduced by $54.6 \%$ in the FnCas 12 a system and more than $38.5 \%$ in the SpCas9 system. We analyzed the changes of VP39 protein expression in the FnCas12a and SpCas9 systems. The Western blot analysis showed that the FnCas12a and SpCas9 systems could significantly inhibit VP39 protein expression. The FnCas12a system only detected the VP39 protein at $48 \mathrm{~h}$ p.i. (Figure 2C). After the FnCas12a system was transfected in BmNSWU1 cells and infected with BmNPV, no significant VP39 protein expression was detected in the FnCas12a system at 0$24 \mathrm{~h}$ p.i.; however, a weak VP39 protein band was detected in the SpCas9 system at $24 \mathrm{~h}$ p.i. The VP39 protein expression of the FnCas12a system was also lower than that of the SpCas9 system at $48 \mathrm{~h}$ p.i. (Figure $2 \mathrm{C}$ ). These results demonstrated that the antiviral ability of the FnCas12a system was more effective than that of the SpCas9 system for BmNPV at the same target site.

\section{Gene-Editing Efficiency of CRISPR/Cas9 and CRISPR/Cas12a Systems in Transgenic Silkworm}

To compare the genome-editing efficiency of CRISPR/Cas12a and CRISPR/Cas9, we constructed FnCas12a and SpCas9 system transgenic vectors. The vectors pBac[IE2-FnCas12aOPIE2-PA-3 × P3 EGFP afm], pBac[U6-gIE1-3 × P3 DsRed afm], and pBac[U6-sgIE1-3 × P3 DsRed afm] expressed the FnCas12a protein, gRNA and the sgRNA target sequence, respectively. The SpCas9 transgenic line was studied previously (Dong et al., 2018a).

After selection of the FnCas12a, gIE1, SpCas9, and sgIE1-positive transgenic lines, double-positive transgenic FnCas12a $\times$ gIE1 and SpCas9 $\times$ sgIE1 lines were obtained through FnCas12a and gIE1 or Cas9 and sgIE1 transgenic line hybridization (Figure 3A). The FnCas12a $\times$ gIE1 line expressed both the FnCas12a protein and gIE1 target sequence, and the SpCas $9 \times$ sgIE1 line expressed both the SpCas 9 protein and sgIE1 target sequence. In the G2 generation, silkworms with both red fluorescent protein and green 

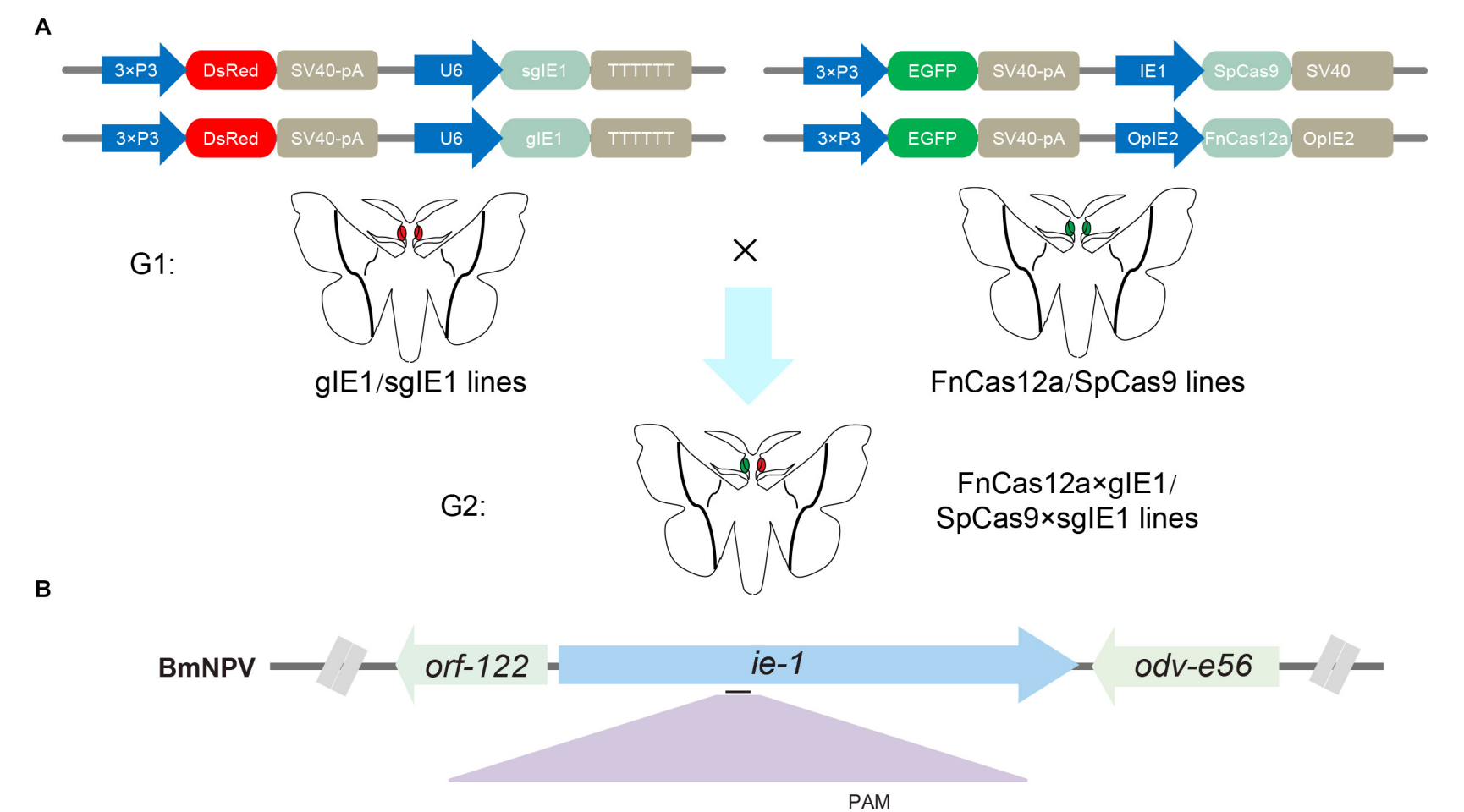

Ref 5'-CCATCGCCCAGTTCTGCTTATAATGCGgAATCTTTTGAGCAGTCTGTTGGTGTGAACCAACCATCGGCAGCTGGAACTAAA-3'

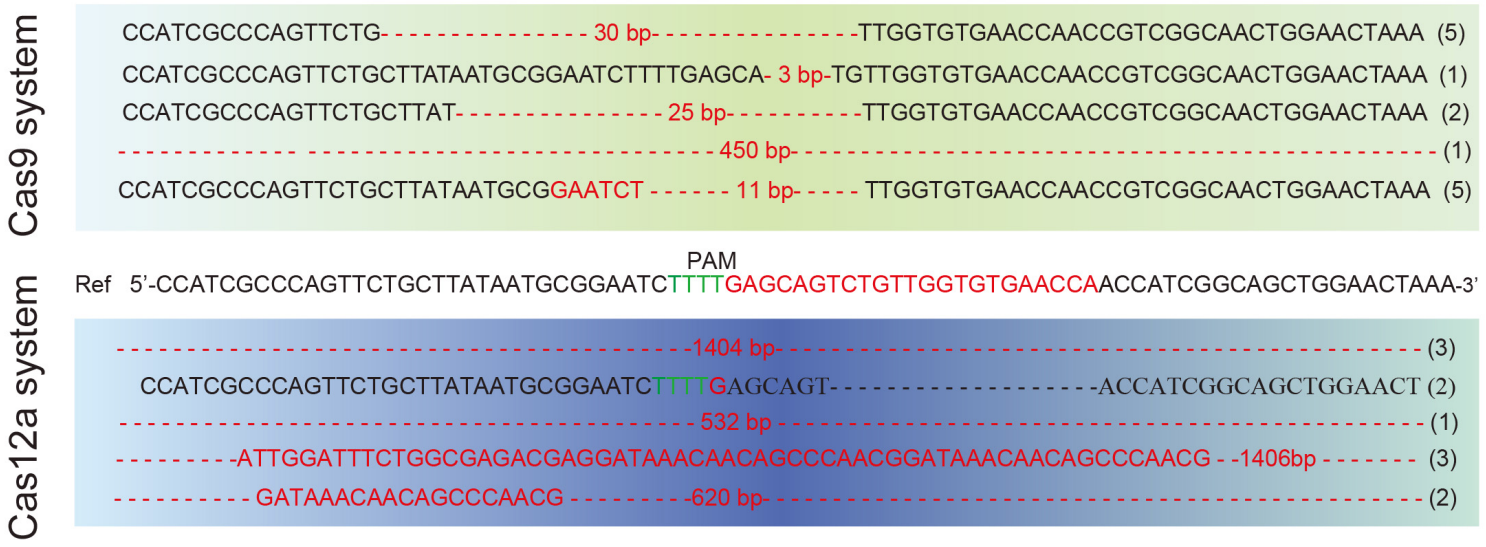

FIGURE 3 | Comparison of gene-editing efficiency of CRISPR/Cas9 and CRISPR/Cas12a systems in transgenic silkworm. (A) Schematic presentation of transgenic vector construction of pBac[OplE2prm-Cas12a-OpIE2-PA-3 $\times$ P3 EGFP afm], pBac[U6-glE1-3 $\times$ P3 DsRed afm], and pBac[U6-sglE1-3 × P3 DsRed afm] (top). Double positive individuals FnCas12a $\times$ glE1 and SpCas9 $\times$ sglE1 obtained were screened by fluorescence microscopy (bottom). (B) Sequencing results of two transgenic lines generated by mutagenesis at ie-1 site.

fluorescent protein expression in their eyes were the doublepositive transgenic FnCas12a $\times$ gIE1 or SpCas9 $\times$ sgIE1 lines (Figure 3A).

To evaluate the potential off-target effects of FnCas12a, we examined all possible off-target sites with high sequence similarity to gIE1 in the silkworm genomes. We selected three non-specific editing sites with the highest similarity for further confirmation by PCR in transgenic lines. Among the three predicted off-targeting sites, we did not detect any off-target mutations in the FnCas12a $\times$ gIE1 transgenic lines (Table 1). These results showed that the FnCas12a systems used in antiviral research had no significant effects on non-specific loci even for editing a highly similar site in the silkworm.

\section{Silkworm Resistance to BmNPV Conferred by the CRISPR/Cas12a System}

To compare the gene-editing efficiency of CRISPR/Cas9 and CRISPR/Cas12a system in transgenic silkworm, we selected the ie-1 gene of BmNPV as the target gene site. The two systems targeted the same site of $i e-1$. After infection with occlusion bodies (OBs) under the same conditions, we determined the 
TABLE 1 | Off-target analysis of CRISPR/Cas9 system in the transgenic silkworm.

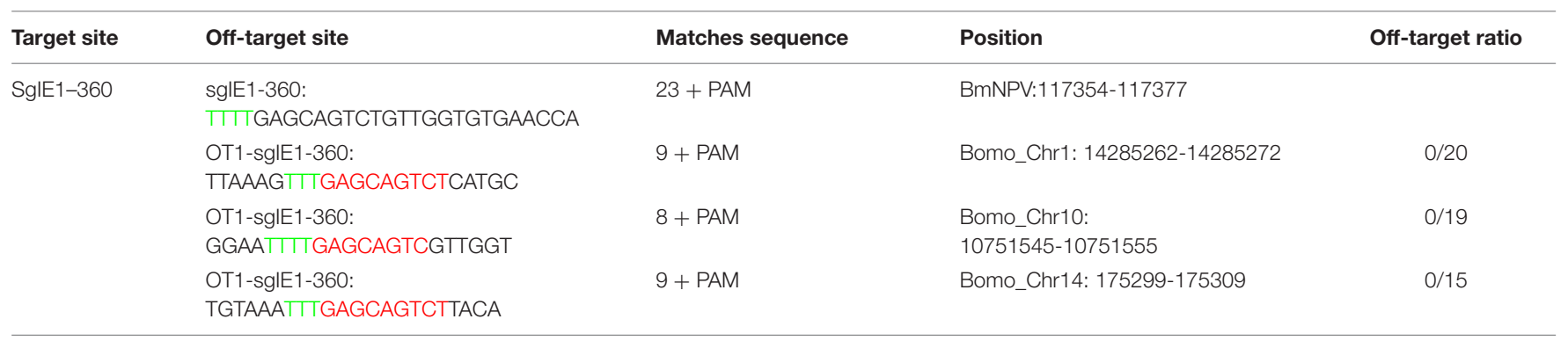

gene-editing efficiency of the target sites in the transgenic hybrid line, FnCas12a $\times$ gIE1 or SpCas $9 \times$ sgIE1. Sequencing of PCR fragments from these lines demonstrated that both the CRISPR/Cas9 and CRISPR/Cas12a gene-editing systems were able to edit the ie-1 gene in the BmNPV genome (Figure 3B). We also found that the sequence of SpCas $9 \times \operatorname{sgIE} 1$ lines was able to edit the target site within the BmNPV genome, which mainly appeared as the absence of 3-30 bp, and only one colony had large deletions in all sequencing (Figure 3B). In contrast, most clones of the transgenic FnCas12a $\times$ gIE1 line showed large deletions, ranging from 500 to $1,400 \mathrm{bp}$. More than $80 \%$ of all sequencings were large deletions (Figure 3B).

We determined whether the FnCas12a system could enhance antiviral activity compared with the SpCas9 system in transgenic lines. The transgenic hybrid lines FnCas12a $\times$ gIE1, SpCas $9 \times$ sgIE1 and the control were infected with $1 \times 10^{6}$ OBs/larva by inoculating 4 fourth instar larvae. Under these conditions, the FnCas12a $\times$ gIE1 and SpCas $9 \times$ sgIE1 lines significantly reduced the BmNPV infection. The survival rate of the SpCas9 $\times$ sgIE1 lines was 59\% until 10-day post infection (d p.i.), whereas the control had large-scale mortality after 5-10 d p.i. (Figure 4A). The survival rate of the FnCas12a $\times$ gIE1 lines was further increased when they were inoculated with OBs. The FnCas12a $\times$ gIE1 lines started to die on $6 \mathrm{~d}$ p.i., but the survival rate of the FnCas12a $\times$ gIE1 lines was still $>65 \%$ after $10 \mathrm{~d}$ p.i. (Figure 4A). These results suggested that the CRISPR/Cas12a system, in transgenic silkworm, could more effectively improve the antiviral activity (Figure 4A). We determined if the surviving transgenic FnCas12a $\times$ gIE1 and SpCas $9 \times$ sgIE1 silkworm lines had altered cocoon characteristics after BmNPV infection. We compared the transgenic lines to the control, and found that they were similar to differences ranging from 11 to 18\% (Figure 4B).

To compare the antiviral ability of different gene-editing systems, we also analyzed the changes of BmNPV gene expression levels at different stages. Similarly, control, FnCas12a $\times$ gIE1 and SpCas9 $\times$ sgIE1 transgenic lines inoculating 4 fourth instar larvae were infected with $1 \times 10^{6}$ OBs/larva. At $0,12,24$, 48, 72, 96, and $120 \mathrm{~h}$ p.i., total RNA was isolated from each transgenic line and the samples were analyzed by real timePCR (RT-PCR). We studied immediate early gene ie-1, early gene $g p 64$, late gene $v p 39$ and very late gene poly of BmNPV to analyze the viral expression levels at different stages. The
RT-PCR results showed that the expression of ie-1, gp64, vp39, and poly genes were maintained at a very low level in the FnCas12a $\times$ gIE1 and SpCas $9 \times$ sgIE1 transgenic lines after BmNPV infection. However, the viral gene expression levels increased as expected in the control (Figure 4C). The viral gene expression levels of FnCas12a $\times$ gIE1 and SpCas $9 \times$ sgIE1 transgenic lines were $10^{4}-10^{5}$-fold lower compared with the control lines. FnCas12a $\times$ gIE1 compared with $\mathrm{SpCas} 9 \times$ gIE1 in different stages was reduced by 10 -fold.

\section{DISCUSSION}

Genome editing has the potential to accurately edit the genomes of model organism (Perez-Pinera et al., 2012; Burgess, 2013; Nakade et al., 2017). Cas9, Cas12a- (Cpf1), Cas12b-, Cas13-, Cas3-, and Cas14- based CRISPR systems have been explored for editing human, animals, plants and microbe genomes (Matsoukas, 2018; Schindele et al., 2018; Moon et al., 2019; Morisaka et al., 2019; Savage, 2019; Shen et al., 2019). Cas12a is a type V CRISPR-effector protein with greater specificity for genome editing in mammals and plants (Fagerlund et al., 2015; Zetsche et al., 2015). To overcome the limitation of Cas9 for antiviral research in B. mori, we engineered an improved CRISPR/Cas12a system and evaluated its efficiency and accuracy for BmNPV genome editing. This application expands the use of CRISPR technology in insect populations.

RNase activity of AsCas12a, FnCas12a, and LbCas12a has been used for genome editing (Fonfara et al., 2016; Kleinstiver et al., 2016; Mahfouz, 2017). AsCas12a has been previously known to efficiently edit insect genomes. However, it was not known which Cas12a system has higher editing efficiency in the Lepidoptera species, such as B. mori. Therefore, we compared the ability of AsCas12a, FnCas12a, and LbCas12a to edit genomes in BmN-SWU1 cells, and demonstrated that the FnCas12a system has potential for use in the development of virus-resistant silkworm lines.

Cas9 system transgenic positive lines can fully edit the target gene (Dong et al., 2018a). To determine the reason for the difference in the antiviral abilities of the Cas9 and Cas12a systems, we constructed transgenic lines. The transgenic FnCas12a $\times$ gIE1 lines could create larger fragment deletions compared with SpCas $9 \times$ sgIE1 lines. Based on the geneediting principles of the Cas9 and Cas12a systems, we believe 


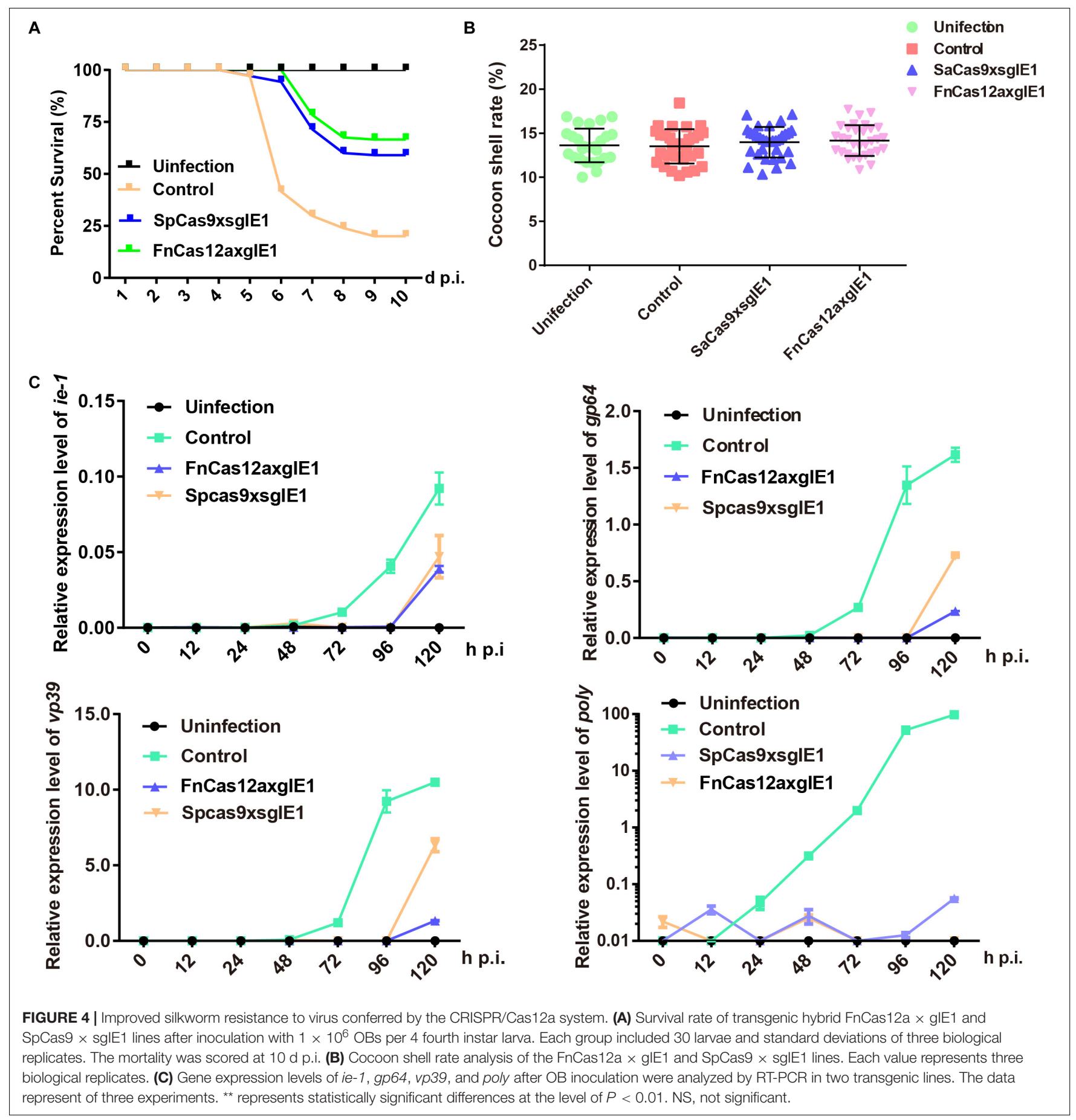

that the cleavage site was distant from the target site of the Cas12a system, and the target site was not destroyed after cleavage (Fagerlund et al., 2015). After the target is cut, the double chain will break, resulting in the deletion of large fragments (Mahfouz, 2017). It also had a greater impact on the function of the viral gene, which could inhibit viral DNA replication. In contrast, the Cas9 system produced blunt ends after editing, and was easily repaired by homologous recombination. The cleavage site of Cas9 was at the target site, resulting in the failure of the system to recognize it again.

Silkworm selection for virus resistance is a traditional method used in the sericulture industry. Interfering with the key genes of BmNPV or overexpression of resistance genes can increase the antiviral ability of the silkworm (Isobe et al., 2004; Subbaiah et al., 2013; Wang et al., 2013). The CRISPR/Cas9 geneediting system has allowed us to improve the antiviral ability of transgenic silkworms. This is accomplished by editing the 
virus early transcriptional activators, editing multiple target sites and genes editing, and editing host-dependent factors (Smith and Johnson, 1988; Dong et al., 2018b). Increased antiviral ability, using traditional means, has reached a limit, and new technology is needed further to increase the resistance to virus attack. We used three different Cas12a systems for editing the BmNPV genome, and screened a Cas12a system with high antiviral ability and gene-editing efficiency in B. mori. This research demonstrated that the antiviral ability of the Cas12a system can be improved compared with the Cas9 system under the same target site in transgenic silkworms (Figure 4A). The Cas12a system can drive many crRNAs through a U6 promoter. In further research we can edit the BmNPV genome through multiple genes and multiple target sites. This will increase the negative effects on the BmNPV genome and improve the virus resistance of transgenic silkworms. We can also try to edit multiple silkworm viruses by synthesizing more crRNAs (such as crRNAs of B. mori densovirus, B.mori cytoplasmic polyhedrosis virus and other infectious diseases of $B$. mori) to one vector. This will further expand the scope and efficiency of transgenic antiviral breeding.

\section{CONCLUSION}

We developed a novel CRISPR nuclease platform, AsCas12a, FnCas12a, and LbCas12a, which can be used for BmNPV genome editing and breeding of virus-resistant silkworms. Our research data indicated that the CRISPR/Cas12a system is a powerful tool for silkworm selection. The system can be used to improve silkworm virus resistance and also as a way to combat other infectious diseases. The successful application of the CRISPR/Cas12a genome editing system can be used to address diseases in $B$. mori and perhaps other economically important insects.

\section{MATERIALS AND METHODS}

\section{Cells}

A B. mori cell line BmN-SWU1, derived from ovary tissue, was maintained in our laboratory and used in this study (Pan et al., 2010). BmN-SWU1 cell lines were cultured at $27^{\circ} \mathrm{C}$ in TC-100 medium (United States Biological, United States). The medium was supplemented with $10 \%(\mathrm{~V} / \mathrm{V})$ fetal bovine serum (FBS) (Gibco, United States).

\section{Viruses}

A recombinant $\mathrm{BmNPV}$ (vA4 $\left.4^{\mathrm{prm}}-\mathrm{EGFP}\right)$ was constructed and used in this study (Zhang et al., 2014). The baculovirus contained a gene encoding for an EGFP marker gene under the control of the B. mori actin A4 promoter. Budded virus (BV) amplification was performed by infection with BmN-SWU1, and then the BV was harvested at $120 \mathrm{~h}$ p.i. Viral titration was performed using the plaque assay method. Occlusion-derived virus (ODV) amplification was performed using oral inoculation with the wild-type (WT)
Chongqing strain of BmNPV in silkworm larvae. OBs were harvested from the infected hemolymph before the larvae died (Dong et al., 2014).

\section{Silkworm Strains}

The control (normal silkworm strain) and transgenic Cas9 strain of B. mori were maintained in our laboratory (Moon et al., 2019). Silkworm larvae were fed on fresh mulberry leaves and maintained at $25^{\circ} \mathrm{C}$ under standard conditions.

\section{Vector Construction}

To explore whether the CRISPR/Cas12a system could be used for gene-editing in B. mori, wild-type (WT) LbCas12a plasmid; pY016 (pcDNA3.1-LbCas12a, Addgene plasmid \# 69988), AsCas12a plasmid; pY010 (pcDNA3.1-AsCas12a, Addgene plasmid \# 69982); and FnCas12a plasmid, pY004 (pcDNA3.1-FnCas12a, Addgene plasmid \# 69976), were obtained from Addgene (Watertown, MA, United States). AsCas12a, FnCas12a, and LbCas12a fragments were cloned into the pSL1180-IE2 ${ }^{\text {prm }}$-OpIE2-PA vector by digestion with BamH I and Kpn I restriction sites, yielding pSL1180-OpIE2 ${ }^{\mathrm{prm}}$ AsCas12a-OpIE2-PA, pSL1180-IE2 ${ }^{\text {prm }}$-FnCas12a-OpIE2-PA,

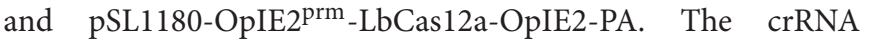
expression cassette under the control of the B. mori U6 promoter was synthesized by BGI and named pSL1180-U6gRNA. The candidate crRNA target sequences were designed using CRISPR design software ${ }^{1}$. We sequentially linked the U6-gRNA expression cassettes into the pSL1180-OpIE2 ${ }^{\text {prm }}$ AsCas12a-OpIE2-PA, pSL1180-OpIE2 ${ }^{\text {prm }}$-FnCas12a-OpIE2-PA, and pSL1180-OpIE2 ${ }^{\text {prm }}$-LbCas12a-OpIE2-PA, and then used restriction enzymes to verify cloning, Cas9 and sgRNA expression cassettes of the target gene ie-1 used previous constructs. We selected the target sites of the BmNPV ie-1 gene as CRISPR/Cas12a and CRISPR/Cas9 gene-editing sites. Sequences for all of the targets of the guide RNAs are provided in Supplementary Table S1.

The transgenic silkworm Cas9 lines were constructed as previously reported. To obtain the green fluorescent protein transgenic vector pBac [OpIE2prm-FnCas12a-OpIE2-PA-3 × P3 EGFP afm], the fragment OpIE2prm-FnCas12a-OPIE2-PA was ligated to the $\mathrm{pBac}[3 \times \mathrm{P} 3 \mathrm{EGFP}$ afm] vector after a single digestion of pSL1180-OpIE2prm-FnCas12a-OpIE2-PA by Asc I restriction endonuclease. Simultaneously, ie-1 target gene vectors pSL1180-U6-gIE1 and pSL1180-U6-sgIE1 were ligated to a pBac [3 $\times$ P3 DsRed afm] vector after single digestion with $\mathrm{Bgl}$ II, which generated a red fluorescent protein transgenic vector for pBac [U6-gIE1 DsRed afm] and pBac [U6-sgIE1$3 \times$ P3 DsRed afm]. All of the primers used are listed in Supplementary Table S1, and all of the constructed vectors were verified by sequencing.

\section{sgRNA and gRNA Design}

BmNPV ie-1 genes were used as targets for gene-editing. To avoid the influence of target sites on the editing efficiency of different gene-editing systems, we chose the same site of ie-1 (located at

\footnotetext{
${ }^{1}$ https://crispr.cos.uni-heidelberg.de/index.html
} 
360 transcription start site of $i e-1)$ as the target site of the Cas12a and Cas 9 gene-editing system. We predicted sgIE1 target gene sequences using an online analysis tool ${ }^{2}$ (Naito et al., 2015). All of the candidate sgRNA target sequences have the GN19NGG sequence. The candidate gRNA target sequences were designed using a CRISPR design software tool ${ }^{1}$ (Stemmer et al., 2015). All of the candidate gRNA target sequences met the requirements of the TTTN PAM recognition domain.

\section{qPCR DNA Replication Assay}

Total DNA was extracted from silkworm cells and larvae using a Wizard Genomic DNA extraction kit (Promega, United States). The copy number of BmNPV was calculated based on quantitative PCR as previously described (Dong et al., 2014). PCR was performed in a $15 \mu \mathrm{L}$ reaction mixture containing using $1 \mu \mathrm{L}$ of extracted DNA solution as the template. All of the experiments were repeated three times.

\section{Western Blot Analysis}

After BmN-SWU1 cells were transfected with the indicated plasmids, the cellular protein was extracted in IP buffer containing $10 \mu \mathrm{L}$ protease inhibitors (PMSF) and boiled for $10 \mathrm{~min}$. Protein suspension samples were separated by $12 \%$ SDS-PAGE and then transferred to a nitrocellulose membrane. The membrane was incubated with mouse $\alpha-\mathrm{HA}$ (1:2,000; Abcam, United Kingdom), mouse $\alpha$-PCNA (1:2000; Abcam, United Kingdom), rabbit $\alpha$-Tubulin (1:5000; Sigma, United States) and rabbit $\alpha$-VP39 (1:2000) for $1 \mathrm{~h}$. Then, the membrane was further incubated with HRP-labeled goat anti-mouse IgG (1:2000; Beyotime, China) and HRP-labeled goat anti-rabbit IgG (1:2000; Beyotime) for $1 \mathrm{~h}$. Finally, the signals on the membrane were visualized by Clarity Western ECL Substrate (Bio-Rad, United States) following the manufacturer's instructions. Tubulins were used to estimate the total protein levels.

\section{Mutagenesis Analysis at Target Sites}

The purified BmNPV genome DNA products were amplified by PCR, and the resulting products were ligated into a pEASY-T5 Zero cloning vector (TransGen Biotech, China). The plasmid was analyzed by Sanger sequencing using M13 primers and aligned with the ie-1 sequence. All of the primers used for detection are presented in Supplementary Table S1.

\section{Microinjection and Screening}

The transgenic vectors pBac [OpIE2prm-FnCas12a-OpIE2-PA$3 \times$ P3 EGFP afm], pBac [U6-gIE1-3 × P3 DsRed afm], and pBac [U6-sgIE1-3 $\times$ P3 DsRed afm] were mixed with the helper plasmid pHA3PIG in the ratio of $1: 1$ and injected into silkworm eggs as previously described (Dong et al., 2018a). The positive individuals were screened by fluorescence microscopy. Double-positive individuals FnCas12a $\times$ gIE1 were obtained by crossing FnCas12a and gIE1, and double-positive individuals

${ }^{2}$ http://crispr.dbcls.jp/
SpCas9 $\times$ sgIE1 were obtained by crossing SpCas9 and sgIE1. All of the positive strains were identified by PCR amplification and fluorescence screening.

\section{Off-Target Assays}

Potential off-target sites in the silkworm genome were predicted using CRISPR design software ${ }^{2}$ (Naito et al., 2015). We screened three potential sites of gIE1 with the highest off-target efficiency and examined them by PCR amplification. The corresponding PCR products were sequenced, and then aligned with the IE1 sequence. All of the off-target site primers used in the study are presented in Supplementary Table S1.

\section{Mortality Analyses}

The OBs of BmNPV were purified from diseased larvae and stored at $4^{\circ} \mathrm{C}$. The transgenic silkworms FnCas12a $\times$ gIE1 and SpCas $9 \times$ sgIE1 were inoculated with $1 \times 10^{6}$ OBs/larva during the fourth instar. Each experimental group contained 30 larvae, and the test was performed in triplicate. Each experimental group was reared individually, and we calculated the survival rate 10 days post-inoculation.

\section{Determination of Expression Levels by RT-PCR}

Total RNA was isolated from each cell or leave, and the cDNAs were synthesized using a cDNA synthesis kit (OMEGA, United States). Gene expression was determined by RT-PCR analysis using an Applied Biosystems 7500 Real-Time PCR System (Life Technologies, United States) with SYBR Select Master Mix Reagent (Bio-Rad). The housekeeping gene (B. mori sw22934) was used as a control. The $2^{-\Delta \Delta C T}$ method was used to calculate the normalized expression of each sample, which was reported as a fold change (Pfaffl, 2001). Three replicates were performed for each reaction. The RT-PCR specific primers are listed in Supplementary Table S1.

\section{Characteristics Analysis of Transgenic Silkworm}

The cocoon volumes of the two transgenic lines, FnCas12a $\times$ gIE1 and SpCas9 $\times$ sgIE1, were analyzed after pupation. Each transgenic line, including 30 larvae, was characterized by the mean of three independent replicates. The cocoon shell rate was calculated as the combined pupa and cocoon weight.

\section{Statistical Analysis}

All of the data are expressed as mean \pm SD of three independent experiments. Statistical analyses were performed with a twosample Student's $t$-test using GraphPad Prism 6. Differences were considered highly significant at $P<0.01$. 


\section{DATA AVAILABILITY STATEMENT}

All datasets generated for this study are included in the article/Supplementary Material.

\section{AUTHOR CONTRIBUTIONS}

$\mathrm{ZD}, \mathrm{QQ}$, and $\mathrm{LH}$ performed the vector cloning, sequencing, cell cultures, and PCR. ZD, QQ, and XZ performed the transgenic injection. JM and $\mathrm{ZH}$ performed the mortality analyses and DNA replication assay. ZD, MP, and CL conceived the experimental design and helped with data analysis. ZD, MP, PC, and CL prepared the manuscript. The final manuscript was reviewed and approved by all authors.

\section{REFERENCES}

Alves-Bezerra, M., Furey, N., Johnson, C. G., and Bissig, K. D. (2019). Using CRISPR/Cas9 to model human liver disease. JHEP Rep. 1, 392-402. doi: 10. 1016/j.jhepr.2019.09.002

Backes, S., Hess, S., Boos, F., Woellhaf, M. W., Godel, S., Jung, M., et al. (2018). Tom70 enhances mitochondrial preprotein import efficiency by binding to internal targeting sequences. J. Cell Biol. 217, 1369-1382. doi: 10.1083/jcb. 201708044

Beretta, M., and Mouquet, H. (2019). CRISPR-Cas9 editing of HIV-1 neutralizing human B cells. Med. Sci. 35, 993-996. doi: 10.1051/medsci/2019196

Burgess, D. J. (2013). Technology: a CRISPR genome-editing tool. Nat. Rev. Genet. 14:80. doi: 10.1038/nrg3409

Chen, S., Hou, C., Bi, H., Wang, Y., Xu, J., Li, M., et al. (2017). Transgenic clustered regularly interspaced short palindromic repeat/Cas9-mediated viral gene targeting for antiviral therapy of Bombyx mori nucleopolyhedrovirus. J. Virol. 91:e02465-16. doi: 10.1128/JVI.02465-16

Dong, D., Ren, K., Qiu, X., Zheng, J., Guo, M., Guan, X., et al. (2016). The crystal structure of Cpf1 in complex with CRISPR RNA. Nature 532, 522-526. doi: $10.1038 /$ nature 17944

Dong, Z. Q., Chen, T. T., Zhang, J., Hu, N., Cao, M. Y., Dong, F. F., et al. (2016). Establishment of a highly efficient virus-inducible CRISPR/Cas9 system in insect cells. Antiviral Res. 130, 50-57. doi: 10.1016/j.antiviral.2016. 03.009

Dong, Z., Dong, F., Yu, X., Huang, L., Jiang, Y., Hu, Z., et al. (2018a). Excision of Nucleopolyhedrovirus form transgenic silkworm using the CRISPR/Cas9 system. Front. Microbiol. 9:209. doi: 10.3389/fmicb.2018.00209

Dong, Z., Huang, L., Dong, F., Hu, Z., Qin, Q., Long, J., et al. (2018b). Establishment of a baculovirus-inducible CRISPR/Cas9 system for antiviral research in transgenic silkworms. Appl. Microbiol. Biotechnol. 102, 9255-9265. doi: 10.1007/s00253-018-9295-8

Dong, Z., Hu, Z., Qin, Q., Dong, F., Huang, L., Long, J., et al. (2019a). CRISPR/Cas9-mediated disruption of the immediate early-0 and 2 as a therapeutic approach to Bombyx mori nucleopolyhedrovirus in transgenic silkworm. Insect. Mol. Biol. 28, 112-122. doi: 10.1111/imb.12529

Dong, Z., Qin, Q., Hu, Z., Chen, P., Huang, L., Zhang, X., et al. (2019b). Construction of a One-vector multiplex CRISPR/Cas9 editing system to inhibit nucleopolyhedrovirus replication in silkworms. Virol. Sin. 34, 444-453. doi: 10.1007/s12250-019-00121-4

Dong, Z. Q., Zhang, J., Chen, X. M., He, Q., Cao, M. Y., Wang, L., et al. (2014). Bombyx mori nucleopolyhedrovirus ORF79 is a per os infectivity factor associated with the PIF complex. Virus Res. 184, 62-70. doi: 10.1016/j.virusres. 2014.02.009

Fagerlund, R. D., Staals, R. H., and Fineran, P. C. (2015). The Cpf1 CRISPRCas protein expands genome-editing tools. Genome Biol. 16:251. doi: 10.1186/ s13059-015-0824-9

\section{FUNDING}

This work was supported by grants from the National Natural Science Foundation of China (Nos. 31902214 and 31872427), Fundamental Research Funds for the Central Universities (Nos. XDJK2020C010 and SWU120008), Natural Science Foundation of Chongqing (cstc2019jcyj-msxm2371), and China Agriculture Research System (No. CARS-18).

\section{SUPPLEMENTARY MATERIAL}

The Supplementary Material for this article can be found online at: https://www.frontiersin.org/articles/10.3389/fbioe. 2020.00841/full\#supplementary-material

TABLE S1 | Sequences of the primers used in this study.

Fonfara, I., Richter, H., Bratovic, M., Le Rhun, A., and Charpentier, E. (2016). The CRISPR-associated DNA-cleaving enzyme Cpf1 also processes precursor CRISPR RNA. Nature 532, 517-521. doi: 10.1038/nature17945

Gao, L., Cox, D. B. T., Yan, W. X., Manteiga, J. C., Schneider, M. W., Yamano, T., et al. (2017). Engineered Cpf1 variants with altered PAM specificities. Nat. Biotechnol. 35, 789-792. doi: 10.1038/nbt.3900

Isobe, R., Kojima, K., Matsuyama, T., Quan, G. X., Kanda, T., Tamura, T., et al. (2004). Use of RNAi technology to confer enhanced resistance to BmNPV on transgenic silkworms. Arch. Virol. 149, 1931-1940. doi: 10.1007/s00705-0040349-0

Kleinstiver, B. P., Tsai, S. Q., Prew, M. S., Nguyen, N. T., Welch, M. M., Lopez, J. M., et al. (2016). Genome-wide specificities of CRISPR-Cas Cpf1 nucleases in human cells. Nat. Biotechnol. 34, 869-874. doi: 10.1038/nbt. 3620

Li, F., Shi, J., Lu, H. S., and Zhang, H. (2019). Functional genomics and CRISPR applied to cardiovascular research and medicine. Arterioscler. Thromb. Vasc. Biol. 39, e188-e194. doi: 10.1161/ATVBAHA.119.312579

Ma, S., Liu, Y., Liu, Y., Chang, J., Zhang, T., Wang, X., et al. (2017). An integrated CRISPR Bombyx mori genome editing system with improved efficiency and expanded target sites. Insect. Biochem. Mol. Biol. 83, 13-20. doi: 10.1016/j.ibmb. 2017.02.003

Mahfouz, M. M. (2017). Genome editing: the efficient tool CRISPR-Cpf1. Nat. Plants 3:17028. doi: 10.1038/nplants.2017.28

Matsoukas, I. G. (2018). Commentary: RNA editing with CRISPR-Cas13. Front. Genet. 9:134. doi: 10.3389/fgene.2018.00134

Moon, S. B., Kim, D. Y., Ko, J. H., and Kim, Y. S. (2019). Recent advances in the CRISPR genome editing tool set. Exp. Mol. Med. 51:130. doi: 10.1038/s12276019-0339-7

Morisaka, H., Yoshimi, K., Okuzaki, Y., Gee, P., Kunihiro, Y., Sonpho, E., et al. (2019). CRISPR-Cas3 induces broad and unidirectional genome editing in human cells. Nat. Commun. 10:5302. doi: 10.1038/s41467-01913226-X

Naito, Y., Hino, K., Bono, H., and Ui-Tei, K. (2015). CRISPRdirect: software for designing CRISPR/Cas guide RNA with reduced off-target sites. Bioinformatics 31, 1120-1123. doi: 10.1093/bioinformatics/btu743

Nakade, S., Yamamoto, T., and Sakuma, T. (2017). Cas9, Cpf1 and C2c1/2/3What's next? Bioengineered 8, 265-273. doi: 10.1080/21655979.2017.12 82018

Pan, M. H., Cai, X. J., Liu, M., Lv, J., Tang, H., Tan, J., et al. (2010). Establishment and characterization of an ovarian cell line of the silkworm, Bombyx mori. Tissue Cell 42, 42-46. doi: 10.1016/j.tice.2009.07.002

Perez-Pinera, P., Ousterout, D. G., and Gersbach, C. A. (2012). Advances in targeted genome editing. Curr. Opin. Chem. Biol. 16, 268-277. doi: 10.1016/j. cbpa.2012.06.007

Pfaffl, M. W. (2001). New mathematical model for relative quantification in real-time RT-PCR. Nucleic Acids Res. 29:e45. doi: 10.1093/nar/29.9.e45 
Savage, D. F. (2019). Cas14: big advances from small CRISPR proteins. Biochemistry 58, 1024-1025. doi: 10.1021/acs.biochem.9b00035

Schindele, P., Wolter, F., and Puchta, H. (2018). Transforming plant biology and breeding with CRISPR/Cas9, Cas12 and Cas13. FEBS Lett. 592, 1954-1967. doi: $10.1002 / 1873-3468.13073$

Shen, W., Zhang, J., Geng, B., Qiu, M., Hu, M., Yang, Q., et al. (2019). Establishment and application of a CRISPR-Cas12a assisted genome-editing system in Zymomonas mobilis. Microb. Cell Fact. 18:162. doi: 10.1186/s12934019-1219-5

Smith, D. B., and Johnson, K. S. (1988). Single-step purification of polypeptides expressed in Escherichia-Coli as fusions with glutathione S-transferase. Gene 67, 31-40. doi: 10.1016/0378-1119(88)90005-4

Stemmer, M., Thumberger, T., Del Sol Keyer, M., Wittbrodt, J., and Mateo, J. L. (2015). CCTop: an intuitive, flexible and reliable CRISPR/Cas9 target prediction tool. PLoS One 10:e0124633. doi: 10.1371/journal.pone.01 24633

Subbaiah, E. V., Royer, C., Kanginakudru, S., Satyavathi, V. V., Babu, A. S., Sivaprasad, V., et al. (2013). Engineering silkworms for resistance to baculovirus through multigene RNA interference. Genetics 193, 63-75. doi: 10.1534/ genetics.112.144402

Wang, F., Xu, H., Yuan, L., Ma, S., Wang, Y., Duan, X., et al. (2013). An optimized sericin-1 expression system for mass-producing recombinant proteins in the middle silk glands of transgenic silkworms. Transg. Res. 22, 925-938. doi: 10 1007/s11248-013-9695-6

Zetsche, B., Gootenberg, J. S., Abudayyeh, O. O., Slaymaker, I. M., Makarova, K. S., Essletzbichler, P., et al. (2015). Cpf1 is a single RNA-guided endonuclease of a class 2 CRISPR-Cas system. Cell 163, 759-771. doi: 10.1016/j.cell.2015. 09.038

Zhang, J., Chen, X. M., Zhang, C. D., He, Q., Dong, Z. Q., Cao, M. Y., et al. (2014). Differential susceptibilities to BmNPV infection of two cell lines derived from the same silkworm ovarian tissues. PLoS One 9:e105986. doi: 10.1371/journal. pone. 0105986

Conflict of Interest: The authors declare that the research was conducted in the absence of any commercial or financial relationships that could be construed as a potential conflict of interest.

Copyright (c) 2020 Dong, Qin, Hu, Zhang, Miao, Huang, Chen, Lu and Pan. This is an open-access article distributed under the terms of the Creative Commons Attribution License (CC BY). The use, distribution or reproduction in other forums is permitted, provided the original author(s) and the copyright owner(s) are credited and that the original publication in this journal is cited, in accordance with accepted academic practice. No use, distribution or reproduction is permitted which does not comply with these terms. 